\title{
Computational and experimental study of probability of galloping and torsional divergence at span structure
}

\author{
Anastasia Fedosova ${ }^{1, *}$, Pavel Churin ${ }^{1}$ and Nikita Ageev ${ }^{1}$ \\ ${ }^{1}$ Moscow state university of civil engineering, Yaroslavskoye shosse, 26, Moscow, Russia, 129337
}

\begin{abstract}
The paper considers study of aeroeslatic stability of span structure using computational and experimental modelling of aerodynamics by wind-tunnel tests and numerical simulation at software package ANSYS CFX. Wind-tunnel test were conducted to to determine steady state wind load coefficients for zero wind attack angle. Using numerical simulation aerodynamic coefficients were obtained for different wind attack angles from -5 to 5 degree with verification to experimental data at zero wind attack angle. Using this approach of combination the accuracy of experimental studies and the convenience of numerical simulation the problems of forecasting phenomena such as galloping and torsion torsional divergence at span structures were solved.
\end{abstract}

\section{Introduction}

Originally, all the phenomena of aerodynamic instability first of all were associated with the aircraft wings, subsequently it turned out that the same phenomena would be characteristic not only of aircraft structures, but also of bridges. The problem of aerodynamic stability of bridge structures came to the fore in 1940, when at a relatively low wind speed $(\mathrm{v}=19 \mathrm{~m} / \mathrm{s})$ for 1 hour the suspension bridge across the river Tacoma with span $855 \mathrm{~m}$ collapsed. The Tacoma catastrophe revealed inadequate structural checks for resistance to wind loads. Destruction of the bridge contributed to research in the field of aerodynamics and aeroelasticity of structures and changes in approaches to the design of all large-span bridges in the world since the 1940s.

Since the Tacoma catastrophe, at designing bridge structures a particular place is given to wind loads, as the span structures have an increased sensitivity to wind forces and the interaction of the span structure with the wind flow can lead to a number of aeroelastic phenomena, the most dangerous are: flutter, buffeting, eddy shedding resonance, parametric resonance and torsional divergence.

In this paper it will be considered two types of aeroelastic phenomena appearing in span structures: galloping and torsional divergence. The acting regulatory documents indicate the need for span structures of complex structures during testing for aerodynamic stability to use the results of testing models of structures in wind tunnels, but such tests are

* Corresponding author: mgsu@,broll.ru 
laborious and expensive. With the modern development of the mathematical apparatus, numerical methods and bases of commercial licensed software an alternative to physical modelling in a wind tunnel can become the transfer of the wind tunnel to a virtual environment with the replacement of the real purging of the model with a virtual one. In this paper, a computational and experimental approach will be applied to simulate aerodynamics, that approach could combine the accuracy of experimental studies and the convenience of numerical simulation.

\section{The object of investigation}

In the paper, a six-lane bridge with a length of $600 \mathrm{~m}$ with a three-meter pedestrian sidewalk provided on it for public transport, located in the Moscow city (Figure 1) is being investigated.

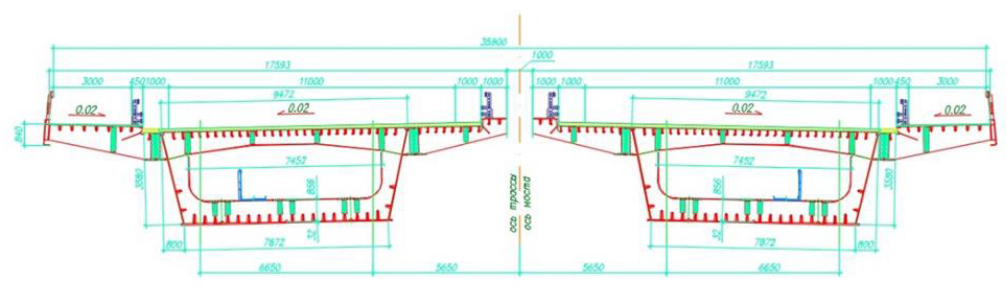

Fig. 1. Typical cross-section of the bridge.

To investigate the possibility of galloping and torsional divergence effects, it is required to determine steady state wind load coefficients: coefficient of drag (Cdrag), coefficient of lift (Clift) and coefficient of pitching moment $(\mathrm{Cmz})$ for different wind attack angles from $-5^{\circ}$ to $5^{\circ}$. According to the proposed method, aerodynamic coefficients will be determined by experiment for zero wind attack angle (corresponding to direction of flow perpendicular to the span structure) and numerical simulation will be performed for following wind angles: $-5^{\circ},-3^{\circ}, 0^{\circ}, 3^{\circ}$ and $5^{\circ}$ with verification to experimental data at zero wind attack angle.

\section{Physical modelling at wind-tunnel}

During the experimental studies, static tests will be carried out to determine the coefficient value of lift (Clift), drag (Cdrag) and pitching moment (Cmz).

Taking into account the dimensions of the wind tunnel working part (4 $\mathrm{m} \times 2.5 \mathrm{~m})$, the optimum scale layout was chosen as 1:70 on the assumption of flow blockage effect.

If the section model has the required mass and inertia characteristics, which are determined by similarity laws, then the actual operation of the span structure will be similar and can be directly deduced from the model behaviour in the wind tunnel.

The design of the model in conjunction with the matched material provides not only a geometric similarity, but also the correct distribution of masses, corresponding to the natural object. Thus, the geometric places of the mass centres of the model and the natural object are also corresponded. The weight of the model was calculated before its manufacture, and then it was measured and finally adjusted on the stand in the wind tunnel. Addition of extra weight was not required because of the small deviation of the measured value from the calculated one. 
Thus, the projected model (Figure 2) corresponds to the natural object according to the following parameters: geometric similarity; the correspondence of the mass distribution; the correspondence between geometric centres of mass and moments of inertia, which fully meets the requirements of experimental studies [1],[2].

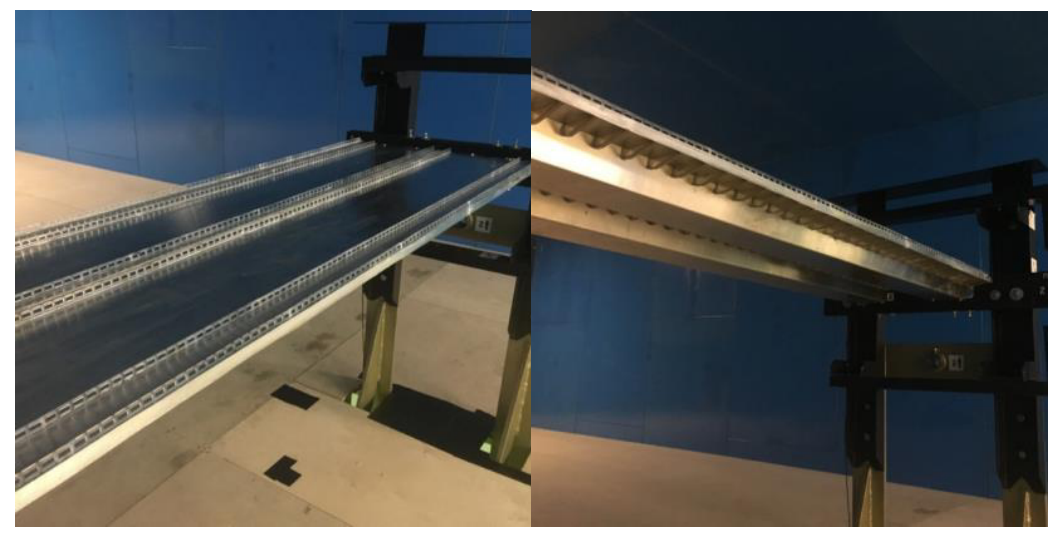

Fig. 2. Model of span structure.

The model was firmly fixed with screws to the 6-component tensors and the lifting force, the drag force and the aerodynamic moment were measured.

The model is exposed to wind, the signals about the oscillations of effort are recorded for $1 \mathrm{~min}$, and then their mean values are calculated. Efforts are expressed in a dimensionless form, called the "aerodynamic coefficient of stability", as it follows:

- coefficient of drag

Cdrag $=\frac{F_{x}}{\frac{1}{2} \rho U^{2} H L} ;$

- coefficient of lift

Clift $=\frac{F_{y}}{\frac{1}{2} \rho U^{2} H L} ;$

- coefficient of pitching moment

$C m z=\frac{M z}{\frac{1}{2} \rho U^{2} B H L}$,

where $\frac{1}{2} \rho U^{2}$ is wind pressure measured in a wind tunnel; $\mathrm{H}=0.053 \mathrm{~m}$ (corresponds to $3.71 \mathrm{~m}$ in full scale), $\mathrm{L}=1,714 \mathrm{~m}$ and $\mathrm{B}=0.254 \mathrm{~m}$ (corresponding to $17.8 \mathrm{~m}$ in full scale) are respectively height, length and width of model.

Aerodynamic coefficients (1), (2) and (3) were measured at different wind speeds to check the dependence of the measured value on the velocity in the wind tunnel. The following values of the aerodynamic coefficients for zero wind attack angle are obtained: Cdrag $=1.2275 ;$ Clift $=0.585 ; \mathrm{Cmz}=0.86237$.

\section{Numerical simulation technology}


Reynolds number, which is characteristic for the problem of the span flow simulation is about 320 thousand, which corresponds to the turbulent motion of gas. The turbulent motion of the air medium near the body is described by the RANS closed with the additional differential relations of the two-parameter dissipative turbulence model.

The instantaneous values of the flow parameters (such as velocity and pressure) are represented as the sum of the averaged quantity and its pulsation component. Thus, in addition to the usual flow parameters (averaged velocity, pressure) additional parameters appear associated with the characteristics of turbulent pulsations. As a result, the system of basic equations of air flow motion is not closed. Closure of the space is accomplished by using additional equations obtained with empirical data. In this case, closure of the space was accomplished by the two-parameter differential model of Menter's turbulence k- $\omega$ SST [3]. The boundary layer is modeled completely turbulent. For the solution a grid method with an implicit finite-difference scheme of conditionally second order (in the region of extrema the first order) approximations for all equations including the equations of the turbulence model was used. The solution was performed using the licensed commercial software package ANSYS CFX, which implements the finite volume method. The established total root-mean-square discrepancies along the entire flow field were about the order of $10^{\wedge}(-4)$. To solve the problem, it took 120 iterations of the solver. The time scale depended on the iteration number according to the sigmoidal law, the final time scale was 0.001 seconds, the characteristic number of iterations to the middle of the height of the sigmoid was 100 , the initial time scale was $10^{\wedge}(-6)$ seconds. For numerical simulation, a computational grid was constructed around the model of the bridge used in the experimental studies. The grid is constructed in the computational domain with a width and length of 6 , a height of 3 and a width of 4.5 meters (Figure 3 ).
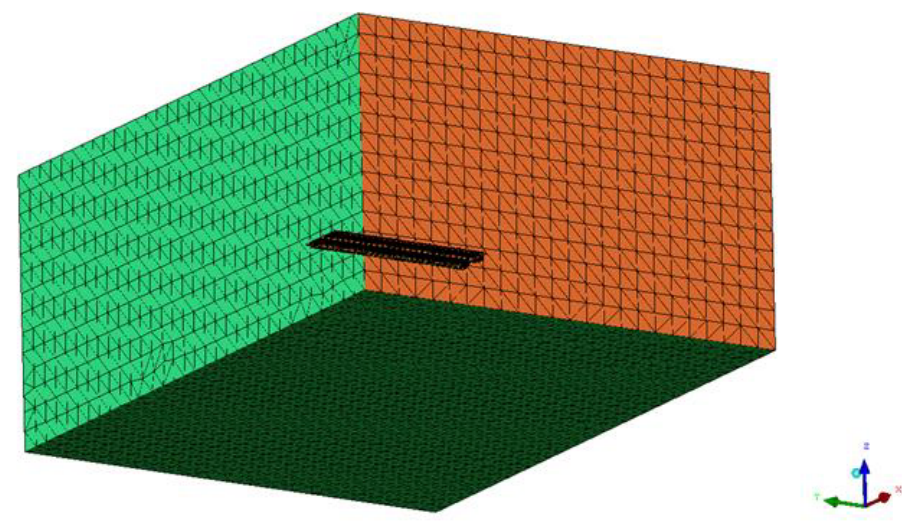

Fig. 3. Computational domain.

In the construction of the computational domain, in the first stage, using the Autoblock method, a surface grid was constructed, consisting mainly of quadrangular elements. Then each quadrangular element was divided into two triangular elements and the grid was checked and corrected. On the basis of the surface mesh, a three-dimensional grid was constructed according to the Delaunay triangulation. At the last stage, the prismatic layers were constructed along the bridge surfaces. In the computational domain at a great distance from the model the maximum size of the final grid element is equal to $0.2 \mathrm{~m}$. On the surfaces of the span structure the characteristic element dimension o is equal to $5 \mathrm{~mm}$. The increase ratio of the elements dimensions is equal to 1.2. The number of prismatic 
layers is 30 . The total number of grid elements is 14 million and 311 thousand. The view of the grid on the span structures surfaces is shown at Figure 4.

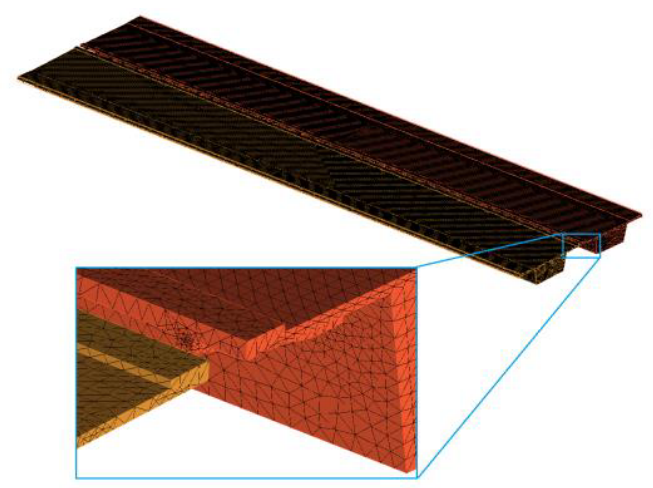

Fig. 4. The calculated mesh for span structure surfaces.

The cross-section of the calculated grid by the plane of symmetry is shown at Figure 5. One can clearly see the sections of the prismatic layers near the surfaces of the bridge. When modelling the flow, the size of the wall cell in the direction perpendicular to the surface is important. This size is characterized by the parameter $\mathrm{Y}+$, which is essentially the Reynolds number calculated from the cell height. If this parameter is much larger than 1 in order of magnitude, the modelling of the boundary layer is performed using so-called wall functions obtained semi-empirically. In the case of $\mathrm{Y}$ +values is the order of 1 , boundary layer modelling is performed using the Reynolds equations directly with the closure of the SST turbulence model.

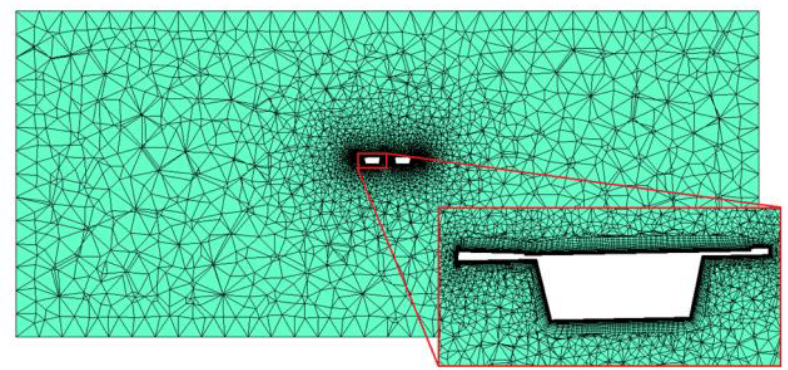

Fig. 5. The cross section of the computational grid by the plane of symmetry.

During the modelling, it is necessary to strive to fulfill the condition $\mathrm{Y}+\sim 1$. The distribution of this parameter over the bridge surfaces is shown at Figure 6. It can be seen that this parameter practically does not exceed 1 on the entire surface of the bridge. The average value is about 0.4 . The maximum value of the parameter $\mathrm{Y}+$ over the entire design area is equal to -2 .

At the input boundary of the computational domain, the flow velocity was set at 20.52 $\mathrm{m} / \mathrm{s}$. The level of turbulent pulsations at the input boundary was set formally because of their rapid repayment as they departed from the boundary and amounted to $1 \%$. On the model surfaces of span structures no-slip condition was set, the flow velocity was zero. On the output boundary (one or two surfaces, as well as for the input boundary) the relative static pressure on average is equal to zero with the possibility of small deviations from the average (Pressure Profile Blend $=0.05$ ). 

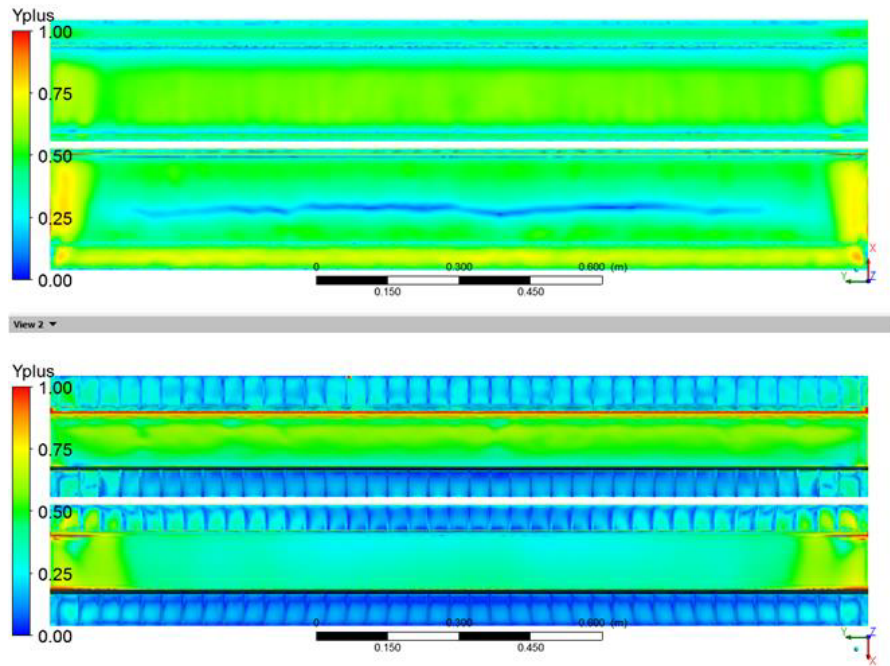

Fig. 6. Distribution of the parameter $Y+$ over the bridge surfaces.

On the side surfaces are open-type conditions (Opening) with a relative static pressure of $0 \mathrm{~Pa}$ and zero gradient of the turbulence parameters, which allows the flow both to enter the design area and to leave it. The initial conditions specified in the calculation are the flow in the computational domain with zero relative static pressure and the velocity corresponding to the input boundary. The obtained values of steady state wind load coefficients for different wind angles are given at Table 1, the value for zero wind attack angle was verified with the experimental results, the error between experimental and numerical simulation was no more than $15 \%$.

Table 1. Wind load coefficients.

\begin{tabular}{|c|c|c|c|c|c|}
\hline $\begin{array}{c}\text { attack } \\
\text { angle }\end{array}$ & Cdrag & Clift & $\mathbf{C m z}$ & $\mathbf{d C m z} / \mathbf{d} \boldsymbol{\alpha}$ & Urk( $\boldsymbol{\alpha}), \mathbf{m} / \mathbf{s}$ \\
\hline $5^{\circ}$ & 1,26 & 2,17 & 0,69 & $-1,30571$ & 177,2 \\
\hline $3^{\circ}$ & 1,32 & 1,84 & 0,78 & $-0,56843$ & 268,6 \\
\hline $0^{\circ}$ & 1,42 & 0,52 & 0,75 & 1,6476 & 1000 \\
\hline$-3^{\circ}$ & 1,41 & $-0,92$ & 0,52 & 5,195764 & 1000 \\
\hline$-5^{\circ}$ & 1,33 & $-1,83$ & 0,34 & 8,30128 & 1000 \\
\hline
\end{tabular}

\section{The study of the bridge to the possibility of galloping}

Galloping is one of the types of aeroelastic instability, most often poorly streamlined flexible elements with aerodynamically unstable cross sections (square or rectangular) are subject to galloping. Galloping is more dangerous than resonance, since it increases with an increase in the flow velocity even above the critical one, and resonance occurs only at a critical wind speed. Under certain flow conditions it is possible that a vertical oscillation of the cross-section with horizontal wind leads to a further self-excitation. In this case, the total damping $d$ for the vertical oscillation is given by 
$d=2 m \xi \omega_{1}+\frac{1}{2} \rho V l\left[C_{L}^{\prime}(0)+C_{D}(0)\right]$,

where $l$ denotes the normalisation length. The first term on the right side, the mechanical damping, is usually positive. Therefore, the criterion for galloping (the so called GlauertDen Hartog) criterion is given by

$\left\lfloor C_{L}^{\prime}(0)+C_{D}(0)\right\rfloor<0$.

If the wind direction is initially not horizontal, the above criterion might be generalized for arbitrary angles $\alpha$.

The figure 7 table holds steady state coefficients for drag, lift and moment dependant on wind attack angle. As can be seen from Figure 8, since the coefficient of lift in the interval increases, the Glauert-Den Hartog criterion stays positive and galloping is impossible. It should be emphasized that this estimate does not allow to determine the actual behaviour of the structure (frequency, amplitude of oscillations), but only establishes the possibility of galloping effect appearance.

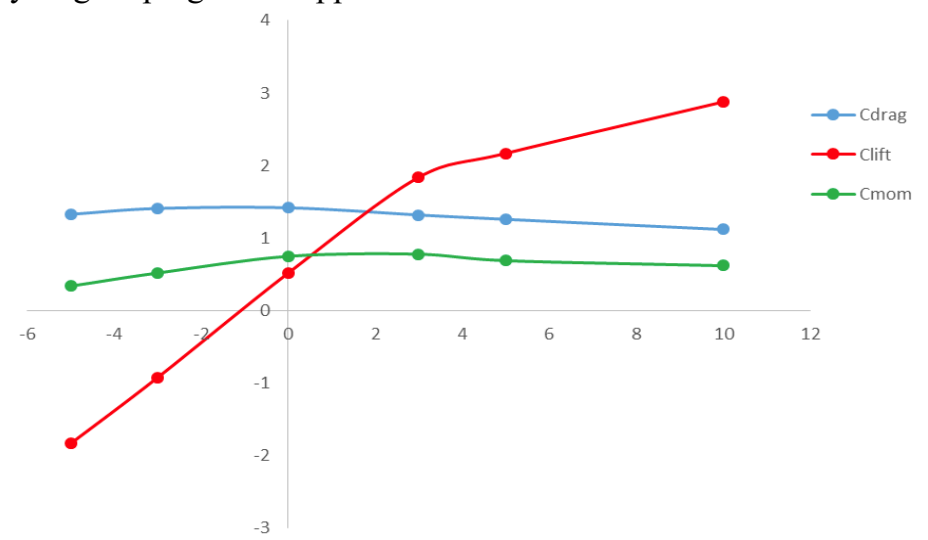

Fig. 7. Drag, lift and moment coefficient dependant on wind attack angle.

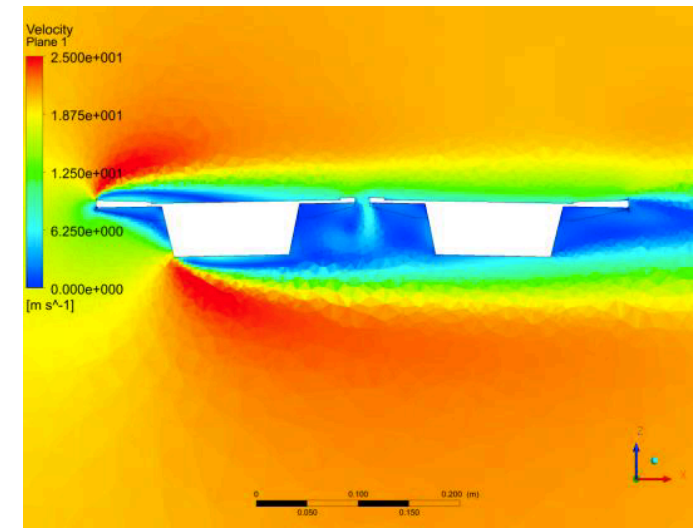

Fig. 8. Bridge in the wind flow.

\section{The study of the bridge to the possibility of torsional divergence}

Torsional divergence is an aperiodic phenomenon of twisting of a span structure up to destruction caused by an increase in the aerodynamic moment, (the moment of elastic 
forces can not balance the moment of aerodynamic forces). The wind speed at which the moment of aerodynamic forces $M(\alpha)$ is equal to the moment of elastic forces $M e$ is called the critical divergence velocity. When this speed is exceeded, the span structure would be destroyed. The critical torsional divergence velocity for small wind attack angles can be calculated by

$U_{c r}(\alpha)=\sqrt{\frac{2 k_{\alpha}}{\rho A L C^{\prime} m z}}$,

where $k_{\alpha}$ denotes the spring constant.

The determination of the critical divergence velocity for different wind attack angles will be carried out by equation 8 , where $k_{\alpha}=1,99 \cdot 10^{5} \mathrm{kNm} / \mathrm{rad}, \rho=1,225 \mathrm{~g} / \mathrm{m}^{\wedge} 3$ and the characteristics of the span structure in full scale are given at paragraph 3 . To determinate the derivatives $d C_{m z}(\alpha) / d \alpha$ following [4] the obtained $\mathrm{Cmz}$ dependence on the wind attack angle is approximated by a polynomial, differentiating which we can find the derivative and use formula (8). The obtained values $d C_{m z}(\alpha) / d \alpha$ and torsional divergence speed in dependency of wind attack angle are given at Table 1 , where speed of $1000 \mathrm{~m} / \mathrm{s}$ represents no torsional divergence; since for these angles of attack $\mathrm{Cmz}$ increases with increasing $\alpha$. The critical wind speed for torsional divergence is about $177 \mathrm{~m} / \mathrm{s}$. To test aerodynamic stability according building code SP 35.13330 .2011 "Bridges and pipes" it is necessary to compare the critical speed of torsional divergence with the maximum wind speed possible in the region of construction. According to the meteorological station located in the construction region, for the ten-year period from 2005 to 2015 the maximum recorded wind speed was $17 \mathrm{~m} / \mathrm{s}$. Taking into account the margin factor for wind load $23.8 \mathrm{~m} / \mathrm{s}$. Thus, since the critical divergence rate $(177 \mathrm{~m} / \mathrm{s})$ is greater than the maximum possible wind speed $(23.8 \mathrm{~m} / \mathrm{s})$ taken with a safety factor of more than 1.5 times, the aerodynamic stability of the span structure in a case of torsional divergence is ensured.

\section{Acknowledgement}

The work was financial supported by the Ministry of Education and Science of the Russian Federation within the framework of the state \#7.6075.2017/BCh, Project «Investigation of the phenomena of aerodynamic instability of building structures in aeroelastic statement, including the development of an innovative methodology for analysing meteorologica data to refine the parameters of the wind load».

All tests were carried out using research equipment of Head Regional Collective Research Centre of Moscow State University of Civil Engineering.

\section{References}

1. P. Churin, O.I. Poddaeva, Aerodynamic testing of bridge structures Applied Mechanics and Materials 477-478, 817-821 (2014)

2. O. Poddaeva, P. Churin, I. Dunichkin, MATEC Web of Conferences 86, 02012 (2016)

3. F.R. Menter, AIAA journal 32.8, 1598-1605 (1994)

4. Yu. L. Rutman, VA Meleshko. Engineering and Construction Journal, 6, 5 - 11 (2011)

5. A.N. Fedosova, International Journal of Applied Engineering Research 10 (20), 4084440845 (2015) 